(C) 2002 Elsevier Science B.V. All rights reserved. Used with permission.

This paper was originally published in the Elsevier Science Journal, Biosensors and Bioelectronics.

Vol. 17, 2002. 71-81. Available at

:http://www.elsevier.com/wps/find/journaldescription.cws_home/405913/description\#description

doi:10.1016/j.physletb.2003.10.071

Keywords: Fluorescence; Lifetime; Energy transfer; Biosensor

\title{
Fluorescence biosensing strategy based on energy transfer between fluorescently labeled receptors and a metallic surface
}

Víctor H. Pérez-Luna ${ }^{\mathrm{a}, 1}$, Saipeng Yang ${ }^{\mathrm{a}}$, Emmanuil M. Rabinovich ${ }^{\mathrm{a}}$, Tione Buranda ${ }^{\mathrm{c}}$, Larry A. Sklar $^{\mathrm{c}}$, Philip D. Hampton ${ }^{\mathrm{b}}$, Gabriel P. López ${ }^{\mathrm{a}, \mathrm{b}}$,

${ }^{a}$ Department of Chemical and Nuclear Engineering, The University of New Mexico, 209 Farris Engineering Center, Albuquerque, NM 87131, USA

${ }^{b}$ Department of Chemistry, The University of New Mexico, 209 Farris Engineering Center, Albuquerque, NM 87131, USA

${ }^{c}$ Cancer Center and Department of Pathology, University of New Mexico, School of Medicine, Albuquerque, NM 87131, USA

\begin{abstract}
A new fluorescence-based biosensor is presented. The biosensing scheme is based on the fact that a fluorophore in close proximity to a metal film $(<100 \AA)$ experiences strong quenching of fluorescence and a dramatic reduction in the lifetime of the excited state. By immobilizing the analyte of interest (or a structural analog of the analyte) to a metal surface and exposing it to a labeled receptor (e.g. antibody), the fluorescence of the labeled receptor becomes quenched upon binding because of the close proximity to the metal. Upon exposure to free analyte, the labeled receptor dissociates from the surface and diffuses into the bulk of the solution. This increases its separation from the metal and an increase of fluorescence intensity and/or lifetime of the excited state is observed that indicates the presence of the soluble analyte. By enclosing this system within a small volume with a semipermeable membrane, a reversible device is obtained. We demonstrate this scheme using a biotinylated self-assembled monolayer (SAM) on gold as our surface immobilized analyte analog, fluorescently labeled anti-biotin as a receptor, and a solution of biotin in PBS as a model analyte. This scheme could easily be extended to transduce a wide variety of protein-ligand interactions and other biorecognition phenomena (e.g. DNA hybridization) that result in changes in the architecture of surface immobilized biomolecules such that a change in the separation distance between fluorophores and the metal film is obtained.
\end{abstract}

\section{Introduction}

Biomolecules labeled with fluorescent dyes are widely used in a variety of scientific and technological applications. A good number of examples can be found in the fields of molecular biology (Skogerboe, 1995; Mifflin, 1996; Gualtieri, 1992; Giuliano et al., 1995), cell biology (Gualtieri, 1992; Giuliano et al., 1995; Dunn et al., 1994; Sabri et al., 1997; Parks and Herzenberg, 1984), biochemistry (Gualtieri, 1992; Giuliano et al., 1995; Wu and Brand, 1994), and medical diagnostics (Skogerboe, 1995; Mifflin, 1996; 
Udenfriend, 1962). These applications are based on the inherent advantages of fluorescent assays, some of which are their high sensitivity (Nie et al., 1994; Nie and Zare, 1997; Xie and Dunn, 1994), the facile introduction of fluorescent labels on a wide variety of biomolecules (such as nucleotides, proteins, and peptides) with minimal effects in their activity (Haugland, 1996; Hermanson et al., 1992), and their usefulness in microscopy applications (Rost, 1992). These characteristics make them valuable tools in several fields of science and technology.

Fluorescent probes for detection of analytes are routinely used in immunoassays. Usually, these require the immobilization of an antibody onto a solid support, which is then exposed to the antigenic analyte and incubated with a labeled reagent (Udenfriend, 1962; Kress-Rogers, 1997). Detection of the bound analyte can be performed by means of introducing labeled analogs of the analyte that compete for the binding sites on the immobilized antibodies (competitive immunoassays) or by introducing secondary antibodies that will bind to the captured antigen (sandwich immunoassay). During the multiple steps involved in these applications, large amounts of labeled antigens or antibodies may be wasted in every assay.

In this work, we develop the basic concept of a reversible, fluorescence-based biosensing scheme based on the sensitivity of the excited state of the fluorophore to its environment. The dependence of fluorescence lifetime on the environment of the excited states has been widely exploited in biosensing applications. As an example, the concentration dependence of fluorescence or phosphorescence emission on dissolved $\mathrm{O} 2$ or $\mathrm{H}+$ ions can be used to monitor changes in $\mathrm{O}_{2}$ based on intensity (Weigl et al., 1994) or lifetime changes (Sacksteder et al., 1993) or pH changes based on intensity (Zhou et al., 1995) or lifetime measurements (Szmacinski and Lakowicz, 1993) in biological systems. However, to our knowledge, the strong dependence between the emission properties of a fluorophore and its separation distance from a metal surface has not been exploited in biosensing applications.

It is well known that metal surfaces strongly influence the fluorescent decay rate of excited molecules placed within close proximity to them. At separation distances between fluorophore and metal surfaces within the order of the emitted wavelength, an oscillatory behavior of fluorescence lifetime with distance is observed (Drexhage, 1974). This is ascribed to constructive and destructive interference effects between the waves directly emitted from the fluorescent molecule and those reflected by the metal surface, which acts like a mirror. However, for distances smaller than about $100 \AA$, radiationless energy transfer to the metal surface dominates the lifetime of the excited molecule (Ritchie and Burstein, 1981). At these short distances, a reduction of the excited state lifetime by several orders of magnitude occurs. Concomitantly, quenching of fluorescence and broadening of the emission spectra can be observed (Cnossen et al., 1993). These phenomena can be exploited to build a sensing device where the fluorophore is in close proximity to the metal surface in the absence of analyte but it separates from the surface upon exposure to the analyte.

A biosensor constructed under this scheme has two advantages: (1) the signal (intensity or lifetime of the excited state) increases with increasing concentrations of the analyte (thus, the baseline for biosensing measurements starts from very small values of intensity or lifetime), and (2) the lifetime of the excited state, which can be used as a 
transduction scheme, is far less sensitive than intensity-based measurements to photobleaching, interference from the background, or changes in the excitation efficiency (i.e. fluctuations in the illumination power density).

One way in which these phenomena can be exploited in biosensing involves the formation of a hapten-antibody (or other receptor/ligand) pair close to a metal surface, with the antibody being tagged with a fluorescent label and the hapten being irreversibly immobilized to the surface of the biosensor. This system is enclosed within a semipermeable membrane that allows diffusion of small analytes (haptens) in and out of the device but keeps the labeled antibodies confined to the space between the metal surface and the membrane. In the absence of analyte in the medium being sampled, the hapten and antibody form a bound complex that is in close proximity to the metal surface. Under these conditions, nonradiative energy transfer to the metal will dramatically affect the fluorescent properties of the labeled antibody (decreased emission intensity and lifetime of the excited state). When the sensing device is exposed to the analyte of interest (unlabeled hapten), the labeled antibody is competitively dissociated from the surface. Upon separation from the surface, the labeled molecule changes its fluorescent properties (increased intensity and lifetime of the excited state). Due to the presence of the semipermeable membrane, the biosensor would reverse when going through cycles of solutions with and without analyte (see Fig. 1).

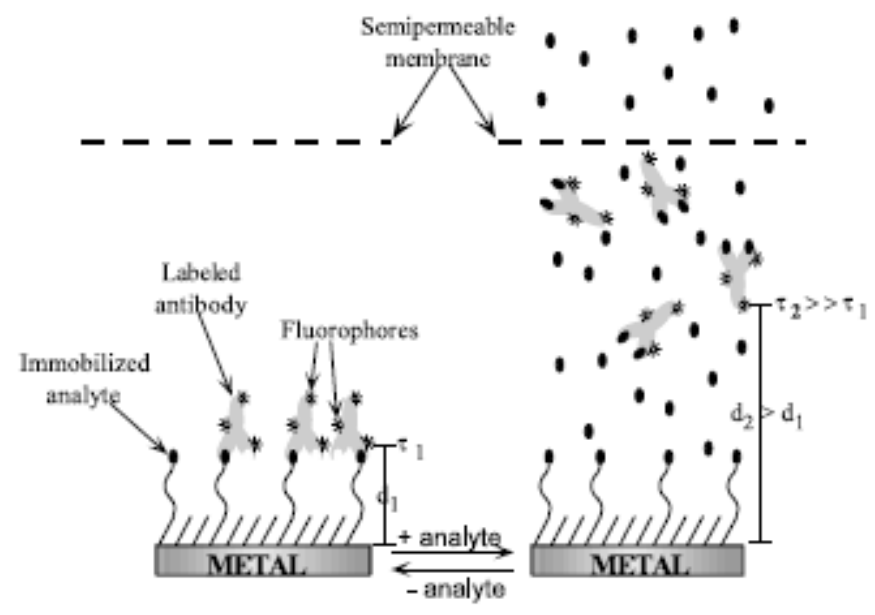

Fig. 1. Molecular biosensing devices based on energy transfer between labeled antibodies and a metal film.

In this work, surface immobilization of the analyte analog is based upon formation of self-assembled monolayers (SAMs) of alkylthiolates on gold films (Ulman, 1991). The attractiveness of this approach resides in the facile creation of surface immobilized receptors on a metal surface (e.g. gold). This provides stable immobilization of the analyte of interest with a well-defined surface chemistry (Ulman, 1991). The facile immobilization of biomolecules into a matrix of fouling resistant groups allows for minimization of non-specific interactions while maintaining specific molecular recognition (Mrksich et al., 1995; Spinke et al., 1993; Pérez-Luna et al., 1999). This minimizes spurious signals and also ensures reversibility of the biosensing device (Mrksich et al., 1995; Spinke et al., 1993; Pérez-Luna et al., 1999). In addition, SAMs allow a controlled separation between the immobilized analyte and the metal film. In fact, 
the thickness of SAMs are precisely in the order needed to dramatically affect the fluorescent properties of surface bound fluorophores (<100 $\AA$ ) (Ulman, 1991).

\section{Experimental}

\subsection{Formation of SAMS}

12-mercaptododecanoic-(8-biotinoylamido-3,6-dioxaoctyl) amide was custom synthesized by Boehringher Mannheim (Penzberg Bayern, Germany), 11-mercapto undecanol was purchased from SIGMA (St. Louis, MO) and used as received. Quartz substrates (G. M. Associates, Inc. \#7525-01) and regular glass slides (Baxter Scientific, \#M6145) were cleaned by immersing them in piranha solution (3/7 by volume of $30 \%$ $\mathrm{H}_{2} \mathrm{O}_{2}$ and $\mathrm{H}_{2} \mathrm{SO}_{4}$ ). CAUTION: piranha solution reacts violently with most organic materials and must be handled with extreme care. They were then thoroughly rinsed with DI water (specific resistance $\sim 18 \mathrm{M} \Omega-\mathrm{cm}$ ), absolute grade ethanol, and blown dried with a stream of nitrogen before mounting them on a rotating carousel in a vacuum chamber for gold deposition. An adhesion layer of $25 \AA$ of chromium (rate of deposition $=0.5 \AA / \mathrm{s}$ ) was deposited on the glass slides before depositing the gold (rate of deposition $=1 \AA / \mathrm{s}$ ) by evaporation of the metals at $10^{-7}-10^{-6}$ Torr. These were monitored with a quartz crystal microbalance thickness monitor. Gold deposited films were immersed overnight in a solution of biotin- and hydroxyl-terminated thiols ( 1 part of biotin-terminated thiol for 19 parts of hydroxyl-terminated thiol on a molar basis) in degassed ethanol. The total thiol concentration for formation of mixed monolayers of hydroxyl and biotin groups was $5 \times 10^{-4} \mathrm{M}$. Under these conditions, the formed SAMs contain a concentration of biotin groups that allow for optimum binding of streptavidin (Spinke et al., 1993; Pe'rez-Luna et al., 1999). After formation of the SAMs, the samples were pulled out of solution; sequentially rinsed with ethanol, DI water, and finally with ethanol again, then dried with a stream of nitrogen, and used immediately. Glass slides with $25 \AA$ chromium and $560 \AA$ gold were used for surface plasmon resonance (SPR) experiments while quartz slides with $25 \AA$ chromium and $2000 \AA$ gold were used for fluorescence measurements.

\subsection{Buffers and proteins}

Dulbecco's phosphate buffered solution and biotin were obtained from SIGMA (St. Louis, MO). Antibiotin, mouse monoclonal 2F5, Alexa 488 labeled IgG was purchased from Molecular Probes (Eugene, OR). Alexa 488 is a fluorescent label with excitation and emission maxima similar to fluorescein but exhibiting remarkably bright, photostable and $\mathrm{pH}$ insensitive fluorescence (Haugland, 1996). Stock solutions of the labeled antibiotin were made in PBS ( $\mathrm{pH} 7.4$ ) at a concentration of $500 \mu \mathrm{g} / \mathrm{ml}$.

\subsection{Surface plasmon resonance (SPR)}

Real time kinetics of binding and dissociation of anti-biotin on biotin-terminated SAMs was determined using a custom made surface plasmon resonance (SPR) instrument equipped with a high precision rotating stage and a flow cell. Measurements were taken with a $633 \mathrm{~nm}$ He_Ne Laser, a BK-7 prism and fixed angle of $70.5^{\circ}$ in a Kretschmann configuration. The details of our SPR instrument are described elsewhere 
(O'Brien et al., 1999). Adsorption of antibiotin onto the biotinylated SAM was performed from a $15 \mu \mathrm{g} / \mathrm{ml}$ solution and dissociation of the antibody from the surface was done in 1 $\mathrm{mM}$ biotin solution in PBS.

\subsection{Fluorescence spectroscopy}

Fluorescence emission spectra and the lifetime of the excited states were obtained with a SPEX Fluorolog 3 instrument (equipped with a Tau-3 system) from Instruments S. A. Fluorescence Division (Edison, NJ). An argon ion laser provided the excitation source with a wavelength of $488 \mathrm{~nm}$. Frequency-domain lifetimefluorescence spectroscopy was used to measure the lifetime of the excited states in our biosensing device. Changes in the phase angle relative to a modulated excitation source concomitant with changes in excited state lifetime of the fluorophores were also measured with a homemade multichannel phase fluorimeter, the details of this instrument can be found elsewhere (Rabinovich et al., 1998).

Measurements of fluorescence were taken with the flat substrate fixed in position with respect to the detector and the laser excitation source throughout the experiment as depicted in Fig. 2. The laser excitation source was incident at $\sim 20-25^{\circ}$ with respect to the surface normal of the sample, while the detector was oriented $\sim 65-70^{\circ}$ with respect to the surface normal of the sample and perpendicular to the laser excitation source. The sample was placed inside a $1 \times 1 \mathrm{~cm}$ quartz cuvette containing PBS buffer and with the monolayer and adsorbed antibodies facing the laser and detectors. This configuration was chosen because it was found to provide for reproducible alignment of the sample with respect to the excitation source and the detector by monitoring the reflection of the laser to a corner in the sample chamber of the spectrofluorometer. It also allowed for a measurable signal to be detected with the sample in a fixed position.

Binding of the fluorescent antibody to the biotinylated surfaces was accomplished by covering the biotinylated substrates with a drop of buffer $(\sim 200 \mu 1)$ followed by inoculation of the drop with the labeled antibody ( $\sim 40 \mu$ of the stock solution). This prevented exposure of the biotinylated surface to the denatured proteins present at the air-liquid interface of the protein solution. After $1 \mathrm{~h}$, the sample was rinsed with buffer and placed inside a quartz cuvette (1-cm path length) equipped with a sample holder to secure the sample in a fixed orientation with respect to the detectors of the spectrofluorimeter (Fig. 2). Dissociation of the antibody from the biotinylated SAM was accomplished by introducing a biotin solution in PBS to a final concentration of $1 \mathrm{mM}$ in the cuvette. In this fashion, dissociation of the antibody from the surface proceeded irreversibly. This was because when biotin was introduced into the cell the dissociated antibody could not rebind to the biotin groups on the surface due to the large excess of biotin in solution. 


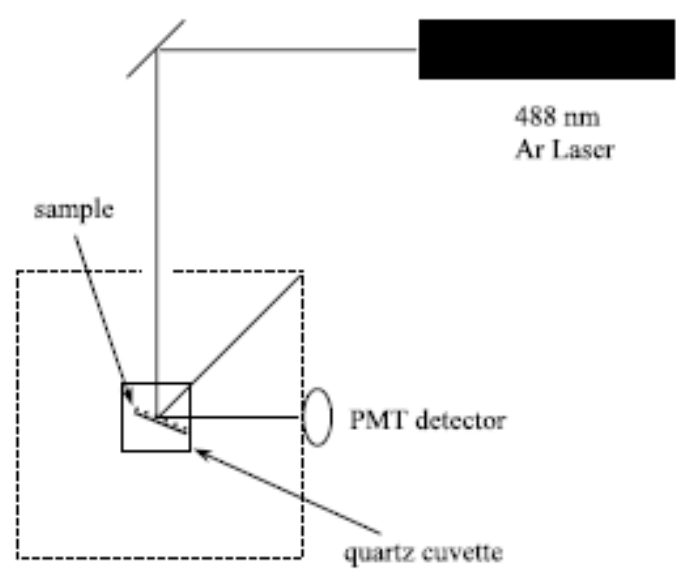

Fig. 2. Experimental setup for emission of fluorescence measurements from flat substrates.

In order to create a reversible biosensor, the Alexa ${ }^{\mathrm{TM}}$ labeled antibody was bound to the biotinylated SAM in a similar fashion. However, after adsorbing and rinsing the excess unbound antibody from the surface, a semipermeable cellulose acetate membrane, Spectra/ Por ${ }^{\circledR} 1$ (Spectrum Laboratories, Inc., Laguna Hills, CA), with a molecular weight cutoff of $8000 \mathrm{Da}$ was placed on the wet surface of the biosensor and securely fixed with a gasket in a quartz flow cell. Background fluorescence from the membrane material was minimized by pretreating it with a $5 \% \mathrm{H}_{2} \mathrm{O}_{2}$ solution under the sunlight for 30 min. After pretreatment, it was thoroughly rinsed with DI water before placing it on the surface of the biosensor.

\section{Results and discussion}

The determination of the response time is important in the design of biosensors. The kinetics of binding and dissociation of anti-biotin from a biotinylated SAM were determined with SPR. Fig. 3a shows the time progression of the binding and dissociation events. Upon introduction of anti-biotin (final concentration in the liquid cell was 15 $\mu \mathrm{g} / \mathrm{ml}$ ), the signal increases quickly until the surface becomes saturated. At this point, the flow cell was flushed with a $1 \mathrm{mM}$ solution biotin in PBS. Competition of free biotin in solution for the biotin binding sites of the antibody caused the anti-biotin molecule to dissociate from the surface. As a result, the signal returned to the initial level because the bound antibody was removed from the SAM. This indicates complete removal of antibiotin from the surface (more than $90 \%$ anti-biotin was removed from the surface in less than an hour). The fact that the bound antibody could be completely competed off the surface with biotin indicates that the antibody was bound to the surface by means of specific molecular recognition between its biotin binding sites and the surface bound biotin of the SAM only. It also shows that nonspecific interactions did not occur between the antibody and the surface neither before nor after binding to the biotinylated SAM (Pérez-Luna et al., 1999). 


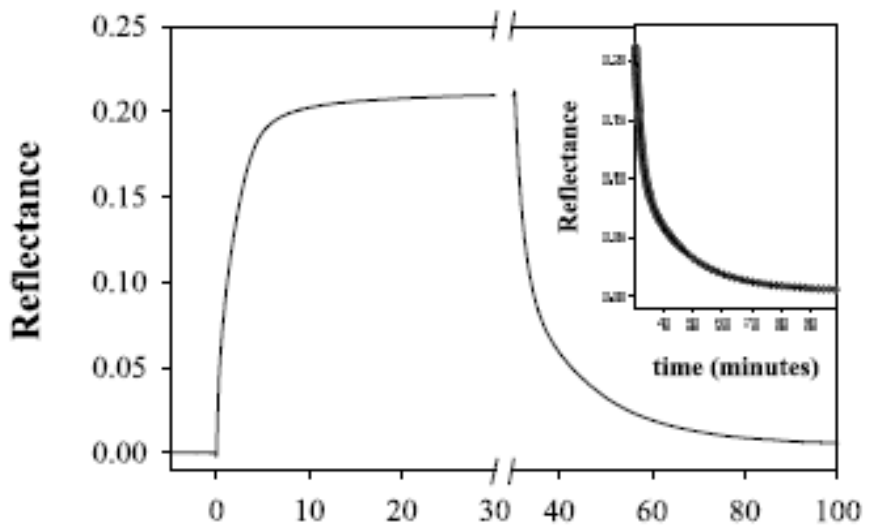

(a)

time (minutes)

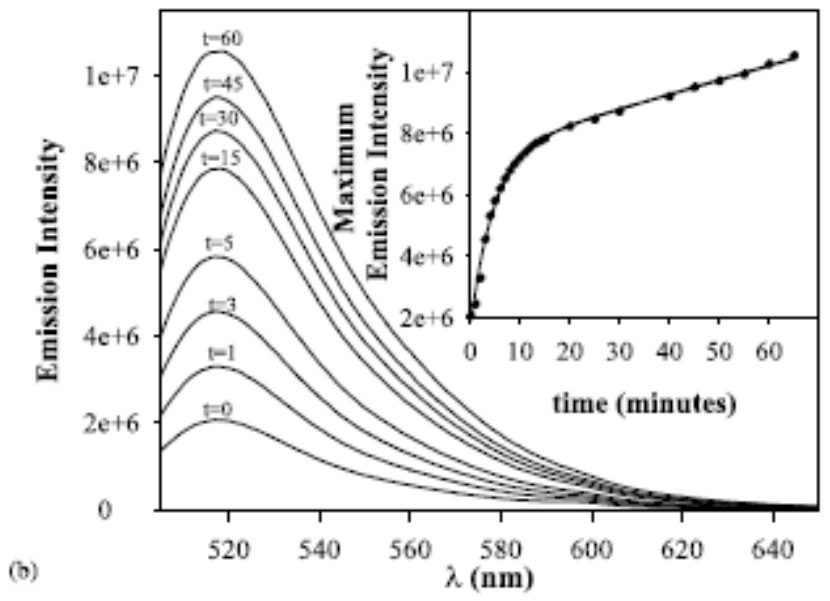

Fig. 3. (A) Binding and dissociation kinetics of anti-biotin $(15 \mu \mathrm{g} / \mathrm{ml})$ on a biotinylated self-assembled monolayer (SAM) as determined by SPR. Measurements taken with a $633 \mathrm{~mm}$ He-Ne Laser, and BK-7 prism and fixed angle of $70.5^{\circ}$ in a Kretschmann configuration. A 1 $\mathrm{mM}$ solution of biotin in PBS was used to competitively dissociate the anti-biotin from the surface. (B) Increased emission of fluorescence upon dissociation of labeled antibody from the surface by free biotin (emission spectra from 505 to $650 \mathrm{~nm}$ at different times after addition of biotin); the inset shows the maximum in the emitted intensity as a function of time.

In the proposed biosensor (Fig. 1) the signal results from changes in the separation distance between the bound antibody and the metal film. These changes occur when the antibody goes from the surface to the bulk solution or vice versa. This involves dissociation of the bound ligand (surface immobilized or in solution) followed by binding of another ligand (soluble or surface immobilized). Since binding is usually much faster than dissociation, especially for proteins interacting with small molecules, we assume that the dissociation process will be the rate-limiting step that determines the dynamic response of the biosensor. The dissociation of the antibody from the biotinylated SAMs follows a double exponential process, which is a result of bivalent interactions with surface bound biotin (Pérez-Luna et al., 1999). The SPR data was thus fit to a double exponential decay by assuming that the reflectance changed linearly with the amount of antibody bound to the biotinylated SAM as described before (Pérez-Luna et al., 1999; Mrksich et al., 1995). The fastest decaying component was assumed to represent the 
process by which a molecule diffuses away from the surface due to dissociation of a single antibody-biotin bond. With this model, the fit of the SPR data to the dissociation process gives a value of $k_{\mathrm{off}}=0.553 \pm 0.002$ per min.

Once the time progression of binding and dissociation of anti-biotin from biotinylated SAMs was known, an experiment to demonstrate the feasibility of our biosensing strategy was carried out. This was accomplished by exposing a biotinylated monolayer on gold with bound anti-biotin (labeled with Alexa ${ }^{\mathrm{TM}} 488$ ) to a $1 \mathrm{mM}$ solution of biotin in PBS buffer. The presence of biotin in solution caused competitive dissociation of the bound IgG (Fig. 3b). Upon dissociation, the IgG molecules diffuse to the bulk and thus, the separation distance between the fluorescently labeled anti-biotin and the gold surface increases (from the surface of the metal to the bulk of the buffer solution). It is well known that the distance where quenching of fluorescence is most dramatic is within the nearest $100 \AA$ from the metal surface (Ritchie and Burstein, 1981). The theoretical length of a fully extended chain of the biotinylated thiol in an all trans configuration and tilted $30^{\circ}$ from the surface normal is $\sim 34 \AA$ (Spinke et al., 1993). Thus, when the antibody is bound to the biotinylated SAM the fluorescence intensity and the lifetime of the excited state are strongly influenced by radiationless energy transfer to the metal. When the labeled antibodies dissociate from the surface, the fluorescent properties of the fluorophores change from being quenched by the metal to being separated to the point where energy transfer to the gold film becomes insignificant. An increased intensity of the emission spectra is thus expected in the presence of biotin as the molecules dissociate to the bulk of the buffer solution. This is indeed the case as shown in Fig. 3b, which shows the emission spectra at varying times after introduction of the biotin solution. From this data, we note that dilution of the antibody into the bulk solution does not offset the increased fluorescence that occurs when the separation distance between the fluorophores and the metal film increases. The inset in Fig. 3 b shows the time progression of the emission intensity corresponding to the maximum in the emission spectra. Fitting the emission intensity data to a double exponential decay that describes the dissociation of the antibody as the rate limiting step gives a value for $k_{\text {off }}=0.22 \pm 0.03$ per min. We note that when fitting this data to a double exponential to estimate the magnitude of $k_{\text {off, }}$ there exists an implicit assumption that the measured change in emission intensity reflects only the kinetics of dissociation of antibodies from the biotinylated surface. This assumption would hold if, on average, the dissociated antibodies diffused into the bulk of the solution following a trajectory normal to the SAM. This situation does not hold in our current experimental setup because the dissociated molecules can diffuse into the bulk solution without ending up facing the metallic surface. That is, they can diffuse to the sides and behind the solid sample (Fig. 2 ). The fact that the value of $k_{\text {off }}$ obtained from emission intensity measurements is about $50 \%$ smaller than the value obtained by SPR could be a result of the dilution effect that occurs when the antibody goes into solution. In other words, when the antibodies are in the surface-bound state the number of fluorescent molecules being excited is larger than when the antibodies dissociate from the surface. Thus, a smaller number of molecules will be in the pathway of the excitation light source after they separate from the surface and become diluted in the cuvette. These two opposite effects affect the signal being measured and the total number of molecules being excited decreases upon dissociation from the surface because of the dilution effects. Due to this, the rate of intensity increase 
observed is smaller than the rate of separation actually taking place in this system. Therefore, the rate constants of dissociation calculated from emission intensity data in our experimental setup will be smaller than the actual value. These effects may also explain the linear trend observed in the emission intensity after $20 \mathrm{~min}$.

An alternative to monitoring emission intensity is the determination of the lifetime of the excited states as our sensor's signal. The advantages of lifetime over intensity measurements were discussed above. The frequency domain measurements before and after dissociation of the Alexa ${ }^{\mathrm{TM}} 488$ labeled antibiotin are shown in Fig. 4a. Before introduction of biotin into our system, the lifetime measured was $0.7 \mathrm{~ns}$, at the end of the experiment, when the labeled antibodies were completed dissociated from the surface, the lifetime had increased to $3.5 \mathrm{~ns}$. These values were obtained by fitting the modulation and phase versus frequency data to a model that assumed the presence of two decaying (lifetime) components. The reported values correspond to the decaying components having the largest contribution to the model.

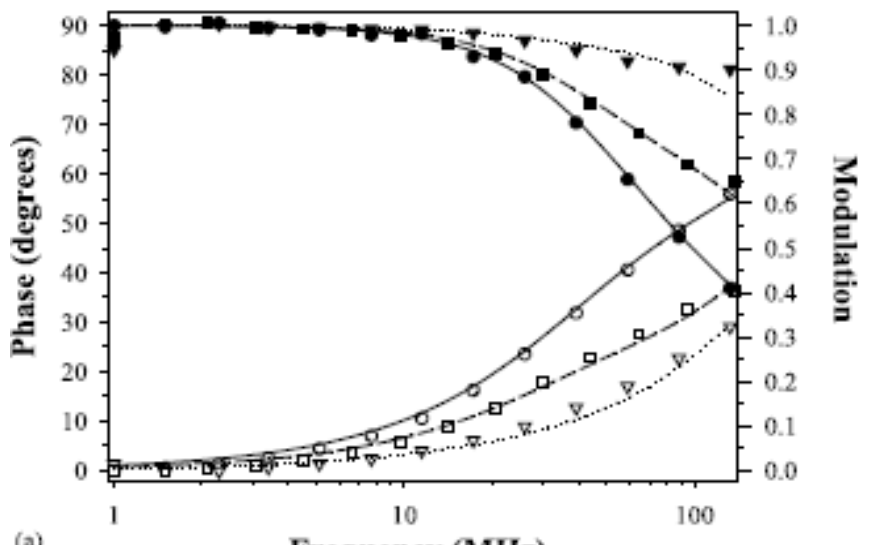

(a) Frequency (MHz)

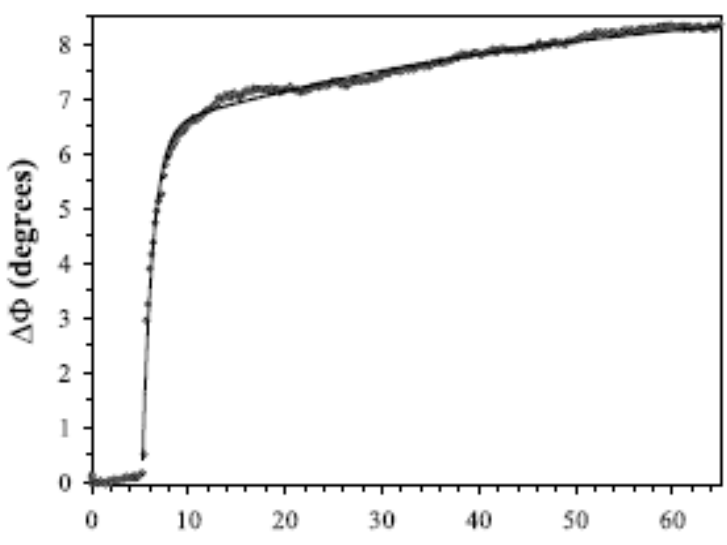

(b)

Time (minutes)

Fig. 4. (A) Frequency domain measurements for determination of lifetimes of the excited states before and after dissociation of antibody from the biotinylated SAM. Open symbols denote the phase angles and filled symbols denote the modulation of the signal as a function of frequency. Circles are data for anti-biotin in solution, squares represent data for biotin treated films and triangles denote anti-biotin on the SAM. The lines represent the fit of a two components model to the frequency-domain data. (B) Change in phase angle (at $40 \mathrm{MHz}$ ) upon exposure of the biosensor to a solution of biotin. 
Although useful, the determination of lifetimes can become cumbersome and time intensive. For example, when more than one decaying component is present in the system (as it would be when a fraction of the labeled antibodies is on the surface and another fraction is in the bulk) the calculation of lifetimes from frequency domain changes in phase and modulation can become computationally intensive. An alternative signal transduction strategy would be to measure the phase angle, at the appropriate frequency, between the emitting fluorophore and a modulated excitation source. Since there is a direct relationship between phase angle and lifetime, this strategy is attractive as a realtime biosensing detection scheme. The measurement of phase changes possesses similar advantages to lifetime over intensity measurements and is illustrated in Fig. 4b, which shows phase changes upon introduction of biotin in solution. This data was acquired using one channel of a multichannel (16 channels) phase fluorimeter (Rabinovich et al., 1998). From this data, an estimate of $k_{\text {off }}=0.39 \pm 0.02$ per min was obtained which was in close agreement to the value found from intensity based measurements. This was calculated by transforming the changes in the phase angle of Fig. $4 \mathrm{~b}$ to conversion assuming a model with two decaying components (Litwiler et al., 1990). The two decaying components were assumed to have lifetimes of 3.5 and $0.7 \mathrm{~ns}$ corresponding respectively to the fluorescence of the antibody in the bulk solution and the antibody bound to the surface as obtained with frequency domain measurements (vide supra). Under this scheme, conversion was defined as the fraction of surface bound antibodies at time zero that detaches and diffuse to the bulk of the solution. A further assumption was that at time zero only surface bound antibodies were present in the system.

To implement the concept of energy transfer to metal surfaces in real-time biosensing applications it is necessary to achieve reversible binding of the labeled antibodies in the system. This requires the removal of free biotin while leaving the labeled antibodies in the system. This was implemented by positioning a semipermeable dialysis membrane over the surface of the biosensor so that the small biotin molecules could diffuse in and out of the system while the larger antibodies become confined to the space between the membrane and the surface of the biosensor. Upon removal of free biotin from the system, the labeled antibodies rebind to the biotinylated monolayer, making the device reversible. Fig. 5 shows the emitted intensities of the biosensing device when it is exposed to alternating cycles of biotin and buffer solutions. Clearly, the maximum fluorescent intensity follows the cycles of buffer and biotin solutions, thus demonstrating the feasibility of the proposed biosensing scheme. In addition, the time needed to rebind the anti-biotin to the biotinylated SAM was comparable to its dissociation from such surfaces. 


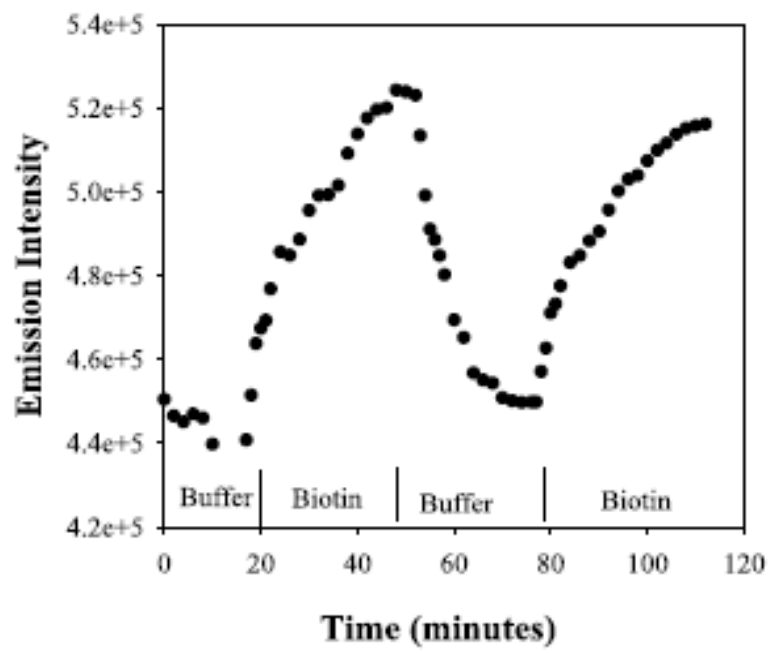

Fig. 5. Emission of fluorescence (at spectral maximum of $518 \mathrm{~nm}$ ) from the biosensor device enclosed in a semipermeable membrane when exposed to alternating cycles of buffer and biotin $(1 \mathrm{mM})$ solution.

\section{Conclusions}

We have shown how radiationless energy transfer between a fluorophore and a metal film can be exploited to make a fluorescence-based, reagentless, reversible biosensor. When the fluorophores are in close proximity to the metal film, their emission intensities become quenched and the lifetime is significantly decreased. Upon dissociation from the metal surface, they diffuse into the bulk of the solution and a significant increase in the emission of fluorescence and the lifetime of the excited state is observed. Proof-ofconcept demonstration of this device was obtained using a biotinylated SAM on gold to which Alexa ${ }^{\mathrm{TM}} 488$ labeled anti-biotin was bound by specific molecular recognition. Upon exposure to free biotin in solution, an increased emission of fluorescence and of lifetime was observed which resulted from the separation of the labeled antibody from the metal film. Reversibility of the biosensor was achieved by confining the labeled antibodies within a small volume in close proximity to the gold film using a semipermeable membrane. The advantages of this device are: (1) a fluorescent signal can be obtained without labeling the analyte being investigated; (2) it has the same advantages of other fluorescent assays and can be implemented to detect changes in intensity, lifetime, or phase shift as the transduction scheme; (3) it is generalizable to any antibody-hapten or protein-ligand pair as long as one of them can be labeled with a fluorophore; (4) it is reversible; reversible; (5) the scheme proposed uses off-the-shelf technology that can be easily implemented.

This general transduction scheme could be extended to transduce a variety of biorecognition events that result in changes of the architecture of the surface immobilized biomolecules or as a way to use metals as platforms to display ligands for fluorescence resonance energy transfer applications. For example, the structure of ssDNA monolayers on gold changes upon hybridization (Levicky et al., 1998). By using neutron reflectivity, it has been found that strands of 5'-HS- $\left(\mathrm{CH}_{2}\right)_{6}$-DNA oligonucleotides chemisorbed on gold change their orientation from lying down on the surface to become oriented toward 
the substrate normal upon hybridization with complementary DNA strands (Levicky et al., 1998). In principle, any change in molecular architecture that significantly perturbs the average distance between a fluorophore, phosphor or other moiety whose luminescence (or other physicochemical) properties are strongly influenced by the metal can be monitored.

\section{Acknowledgements}

This work was funded by grants from the Office of Naval Research (N00014-95-1-1315, N00014-95-1-0901, N00014-95-1-0255), the National Science Foundation (CTS9624841). We thank Michael J. O’Brien for building our surface plasmon resonance instrument.

\section{References}

Cnossen, G., Drabe, K.E., Wiersma, D.A., 1993. Fluorescent properties of submonolayers of rhodamine 6G in front of a mirror. J. Chem. Phys. 98, 5276-5280.

Drexhage, K.H., 1974. In: Wolf, E., (Ed.), Progress in Optics XII, North Holland, Amsterdam.

Dunn, K.W., Mayor, S., Myers, J.N., Maxfield, F.R., 1994. Applications of ratio fluorescence microscopy in the study of cell physiology. FASEB J. 8, 573-582.

Giuliano, K.A., Post, P.L., Hahn, K.M., Taylor, D.L., 1995. Fluorescent protein biosensors: measurement of molecular dynamics in living cells. Annu. Rev. Biophys. Biomol. Struct. 24, 405-434.

Gualtieri, P., 1992. Molecular-Biology in living cells by means of digital optical microscopy. Micron Microsc. Acta 23, 239-257.

Haugland, R.P., 1996. Handbook of Fluorescent Probes and Research Chemicals, Sixth ed. Molecular Probes, Inc, Eugene, OR.

Hermanson, G.T., Mallia, K.M., Smith, P.K., 1992. Immobilized Affinity Ligand Techniques. Academic Press, San Diego, CA.

Kress-Rogers, E. (Ed.), 1997. Handbook of Biosensors and Electronic Noses: Medicine, Food, and the Environment. CRC Press, Boca Raton, FL.

Levicky, R., Herne, T.M., Tarlov, M.J., Satija, S.K., 1998. Using self-assembly to control the structure of DNA monolayers on gold: a neutron reflectivity study. J. Am. Chem. Soc. 120, 9787-9792.

Litwiler, K.S., Huang, J., Bright, F.V., 1990. Effects of inaccurate reference lifetimes on interpreting frequency-domain fluorescence data. Anal. Chem. 62, 471-476.

Mifflin, T.E., 1996. Recent developments in molecular diagnostic assays for genetic-diseases. J. Clin. Ligand. Assay 19, 27-42.

Mrksich, M, Grunwell, J.R., Whitesides, G.M., 1995. Biospecific adsorption of carbonic-anhydrase to selfassembled monolayers of alkanethiolates that present benzenesulfonamide groups on gold. J. Am. Chem. Soc. 117, 12009-12010.

Nie, S.M., Chiu, D.T., Zare, R.N., 1994. Probing individual molecules with confocal fluorescence microscopy. Science 266, 1018-1021. 
Nie, S.M., Zare, R.N., 1997. Optical detection of single molecules. Ann. Rev. Biophys Biomol. Struct. 26, $267-296$.

O’Brien, M.J., Brueck, S.R.J., Pérez-Luna, V.H., Tender, L.M., López, G.P., 1999. SPR biosensors: simultaneously removing thermal and bulk-composition effects. Biosens. Bioelectr. 14, 145-154.

Parks, D.R., Herzenberg, L.A., 1984. Fluorescence-activated cell sorting: theory, experimental optimization, and applications in lymphoid cell biology. Methods Enzymol. 108, 197-241.

Pérez-Luna, V.H., O’Brien, M.J., Opperman, K.A., Hampton, P.D., López, G.P., Klumb, L.A., Stayton, P.S., 1999. Molecular recognition between genetically engineered streptavidin and surfacebound biotin. J. Am. Chem. Soc. 121, 6469-6478.

Rabinovich, E., O’Brien, M.J., Srinivasan, B., Elliot, S., Long, X.C., Jain, R., Pérez-Luna, V., Zhou, Y., Tender, L., López, G., 1998. Compact LED-based phase fluorimeter detection system for chemical and biosensor arrays. Proc. SPIE 3258, 2-10.

Ritchie, G., Burstein, E., 1981. Luminescence of dye molecules adsorbed at a Ag surface. Phys. Rev. B 24, 4843-4846.

Rost, F.W.D., 1992. Fluorescent Microscopy, vol. 1 and 2. Cambridge University Press.

Sabri, S., Richelme, F., Pierres, A., Benoliel, A.M., Bongrand, P., 1997. Interest of image processing in cell biology and immunology. J. Immunol. Methods 208, 1-27.

Sacksteder, L.A., Demas, J.N., Degraff, B.A., 1993. Design of oxygen sensors based on quenching of luminescent metal-complexes: effect of ligand size on heterogeneity. Anal. Chem. 65, 3480-3483.

Skogerboe, K.J., 1995. Molecular biology techniques. Anal. Chem. 67, R449-R454.

Spinke, J., Liley, M., Schmitt, F.J., Guder, H.J., Angermaier, L., Knoll, W., 1993. Molecular recognition at self-assembled monolayers: optimization of surface functionalization. J. Chem. Phys. 99, 7012-7019.

Szmacinski, H., Lakowicz, J.R., 1993. Optical measurements of pH using fluorescence lifetimes and phasemodulation fluorometry. Anal. Chem. 65, 1668-1674.

Udenfriend, S., 1962. Fluorescence Assay in Biology and Medicine. Academic Press, New York.

Ulman, A., 1991. An Introduction to Ultrathin Organic Films: from Langmuir-Blodgett to Self Assembly. Academic Press, Boston, MA.

Weigl, B.H., Holobar, A., Trettnak, W., Klimanti, I., Kraus, H., Oleary, P., Wolfbeis, O.S., 1994. Optical triple sensor for measuring $\mathrm{pH}$, oxygen, and carbondioxide. J. Biotechnol. 32, 127-138.

Wu, P.G., Brand, L., 1994. Resonance energy transfer: methods and applications. Anal. Biochem. 218, 113 .

Xie, X.S., Dunn, R.C., 1994. Probing single molecule dynamics. Science 265, 361-364.

Zhou, Y., Marcus, E.M., Haugland, R.P., Opas, M., 1995. Use of a new fluorescent-probe, seminaphthofluorescein-calcein, for determination of intracellular $\mathrm{pH}$ by simultaneous dual-emission imaging laser-scanning confocal microscopy. J. Cell. Physiol. 164, 9-16. 